\title{
Design and Implementation of Marker less Augmented Reality Application for Cockroach Phobia Therapy Using Adaptive Threshold
}

\author{
Fiandra Fatharany, Anny Yuniarti, and Ridho Rahman Hariadi. \\ Department of Informatics, Faculty of Information Technology, Institut Teknologi Sepuluh Nopember (ITS) \\ Jl. Arief Rahman Hakim, Surabaya 60111 Indonesia \\ e-mail: anny@if.its.ac.id, ridho@if.its.ac.id, fatharany11@mhs.if.its.ac.id
}

\begin{abstract}
Augmented reality (AR) technology is useful for treating several psychological problems, including phobias such as fear of flying, agoraphobia, claustrophobia, and phobia to insects and small animals. However, the currently existing applications for therapy of cockroach phobia that uses AR technology are still very dependent towards the presence of markers, which might lessen the feeling of being in an actual scenario from everyday lives.
\end{abstract}

In this paper, we created a system that is able to use everyday things as a replacement for markers for phobia therapy for cockroach. There are five main processes: getting the live streaming feed from camera, preprocessing, extracting the center point of the objects, tracking the marker-substitute objects, and lastly, instantiating cockroaches randomly after user lifts the objects according to the number and mode of the cockroaches, whether it is moving or not, that are predetermined by the user.

The evaluation in this paper includes eight participants that are carefully selected based on their Fear of Spiders Questionnaire (FSQ) score that is translated into Indonesian and modified to accommodate cockroaches instead of spiders. The results is that the system can induce anxiety level on participants with the highest score of 10 , which is the highest score in Standard Unit of Discomfort scale (SUDs). While the presence and reality judgment of this paper has the highest score of 7 which is also the highest score in Slater-Usoh-Steed Questionnaire (SUS).

Keywords-adaptive threshold, augmented reality, marker less, phobia therapies for cockroaches.

\section{INTRODUCTION}

$\mathrm{P}_{\mathrm{p}}$ HOBIA as a special form of fear which (a) is out of proportion to the demands of a situation; (b) cannot be explained or reasoned away; (c) is beyond voluntary control; and (d) leads to avoidance of the feared situation [1]. Subsequently, Martin \& Pear [2] and Smith [3] also stated that phobia is an intense fear of something that are slightly or might not generate real threat.

Augmented Reality (AR) consists of an environment in which various virtual elements are incorporated into the user's perception of the real world [4]. The main objective of the application is to enhance the user's perception of an experience in the real world, wherein 3D virtual objects appear to coexist with real elements in the actual world [5], [6]. Significantly, the virtual elements in AR add relevant and helpful information to the real scene [7].

Case study by M. Carmen Juan and Dennis Joele stated if the markers were not visible, the patient would not know that the animals were going to appear, and when they did appear, they would produce the desired surprise [8], [9]. Therefore, it would induce a higher level of anxiety and able to mimic the actual scene from a real life experience.

This marker less augmented reality application for cockroach phobia therapy is using adaptive threshold method for its image processing and tracking marker-substitute objects. The objects that we used are ordinary objects that existed in everyday lives. The model of cockroaches [10] instantiated according the object's center point. We used an external camera to capture the testing environment consists of marker-substitute objects and plain background.

This paper made with a purpose to create an application that would be able to accommodate a therapy for cockroach phobia where participants encounter and search the feared cockroaches in the same way they would do so in their everyday lives.

\section{METHOD}

\section{A. Data Extraction}

This study used Unity [11] as a platform to develop this application. WebCamDevices function would get the entire available camera connected to the computer. To get the live feed from external camera, we used WebCamTexture function.

\section{B. Preprocessing}

1) Adjusting the Orientation of Input

The image shown on the screen need an adjustment to match the orientation of the testing environment. Thus, we used flip function from class Core with the parameter of -1 to flip the input image based on $\mathrm{x}$-axis and $\mathrm{y}$-axis using OpenCV library [12].

2) Converting the Image into Black and White

This process used cvtColor function with the parameter of COLOR_BGR2GRAY using OpenCVForUnity library [13] because for the next step, adaptive threshold, cannot process an RGB image.

\section{3) Adaptive Threshold}

Adaptive threshold is one of the image processing function provided by OpenCVForUnity library. For this step, we used Binary as its thresholding type and Gaussian as its adaptive method. The binary thresholding type was used to transform the RGB image into binary with the color black for the objects 
and white for the background as can be seen on (1) where $\operatorname{src}(\mathrm{x}, \mathrm{y})$ is the input and $\mathrm{T}(\mathrm{x}, \mathrm{y})$ is the thresholding value. Whereas we defined maxValue as 255 which is the value for white. In Gaussian method, $\mathrm{T}(\mathrm{x}, \mathrm{y})$ is a weighted sum of the blocksizexblocksize neighborhood of $(\mathrm{x}, \mathrm{y})$. We defined the blocksize as 33 and 3.5 for constant value $C$.

$$
d s t(x, y)=\left\{\begin{array}{cc}
\max V a l u e & \text { if } \\
0 & \operatorname{src}(x, y)>T(x, y) \\
0 & \text { otherwise }
\end{array}\right.
$$

\section{Extracting the Center Point of the Marker-substitute Objects}

This extraction is crucial for the final step of this application. The output from this stage was the center point of all of the objects.

1) Contour

Contour is a sequence of points that represents a curve in an image whereas each sequence holds an information to where the next point in the curve is. In OpenCVForUnity library, this method is implemented using cvFindContour function. This function will compute the contour of a binary image. The binary image can be taken from cvCanny function or through image processing using cvThreshold or cvAdaptiveThreshold function.

2) Moment

We used moment to compute the center mass point, an area, or every points of every contour in an image. To locate the center point of the marker-substitute objects displayed on screen, we used CvMoments function [14].

3) Draw Contour

The center point of the marker-substitute objects will be shown using drawContour function from OpenCVForUnity library in the form of a small circle and a text with the format of $(\mathrm{x}, \mathrm{y})$.

\section{Tracking the Marker-substitute Objects}

We used Enumerable.Range function to track and determined whether the marker-substitute objects still exist or not within the frame by tracking the center point $(\mathrm{x}, \mathrm{y})$ of the objects. If the center point of the objects did not exist within a range of \pm 15 pixel for 30 frame or 1 second then the cockroach will be instantiated.

\section{E. Instantiating the Cockroach Models}

Once a marker-substitute object was established gone, a number (that are predetermined by the participant) of cockroach model will be randomly shown in the center point of the object. This is to mimic real life situations where participants are in the act of searching underneath their personal belongings.

\section{CASE STUDY}

\section{A. Testing Environment}

The application runs on Intel ${ }^{\circledR}$ Core $^{\mathrm{TM}}$ i5-3317U CPU @ $1.70 \mathrm{GHz}, 1.70 \mathrm{GHz}$ processor with $4 \mathrm{~GB}$ memory and operating system of Microsoft Windows Embedded 8 Pro 64bit and using an external camera with the resolution of $640 \times 480$, video format 24 bit $R G B$, and frame rate $320 \times 24$ up to $30 \mathrm{frame} / \mathrm{sec}$.

\section{B. Measures}

1) Measuring the participants' level of fear towards cockroaches using the modified and translated Fear of Spiders Questionnaire (FSQ) [15] as used in the scientific journal by J. Breto'n-Lo'pez, Ph.D [7].

2) During the immersion, participants must rate their maximum level of anxiety on a 0 -to- 10 scale, where 0 is no anxiety and 10 is high anxiety for each scenarios using the translated Subjective Units of Discomfort scale (SUDs) [16].

3) After using the application, participants filled in a modified and translated Slater-Usoh-Steed Questionnaire (SUS) [17] to measure the reality and presence judgment with the scale of 1-7.

\section{Participants}

Seven males and six females participated in the study. Their age ranged from 20 to 23 years. All of the participants are students of a higher education. In order to be included in the study, we considered the following criteria: having the total scores over 40 in their FSQ based on their initial interview whether they are disgusted and/or scared of cockroach or not. Only six males and two females are eligible to join the study. Participants read and signed a consent form accepting the study they were going to participate in and agreeing to let us use their data in the study.

\section{Procedure}

The procedure of this study used the procedure in case study by M. Carmen Juan and Dennis Joele [9] as a guideline. The study performs following steps:

1) We explained the direction on how to use the application to the participants.

2) During the study, participants are required to look at the computer screen while lift one of the marker-substitute objects to search for cockroaches as can be seen on Fig. 1. That consists of several scenarios:

a. One static cockroach.

b. One moving cockroach.

c. Three static cockroaches.

d. Three moving cockroaches.

3) After the immersion, participants filled in the SUS questionnaire and the study finished. The total time was about 10 to 15 minutes for each individual.

\section{RESULTS}

For the anxiety level, Table 1 shows the result related to the ability of the application to induce anxiety in participants. The result shows that this application may only induced a small level of anxiety in each participants with the highest score of 7 and the lowest is 1 for scenario one that shows one static cockroach, whilst for scenario two that shows 1 moving cockroach has the highest score of 8 and the lowest score of 1 . As for scenario 3 , which has three static cockroaches, shows a higher mean of scores with the highest score is 8 and the lowest score is 2. Meanwhile, scenario 4 that shows three 


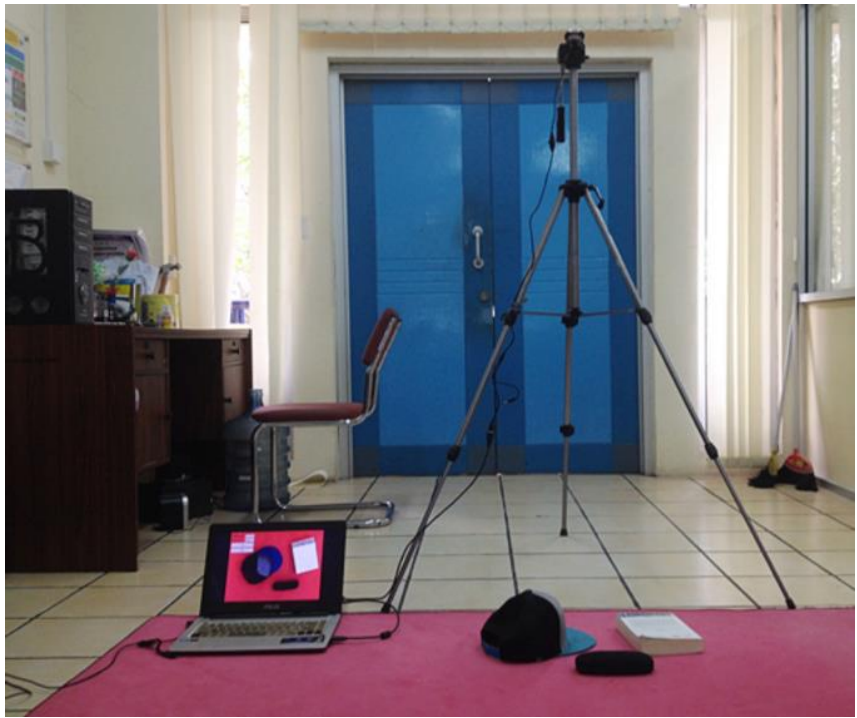

(a)

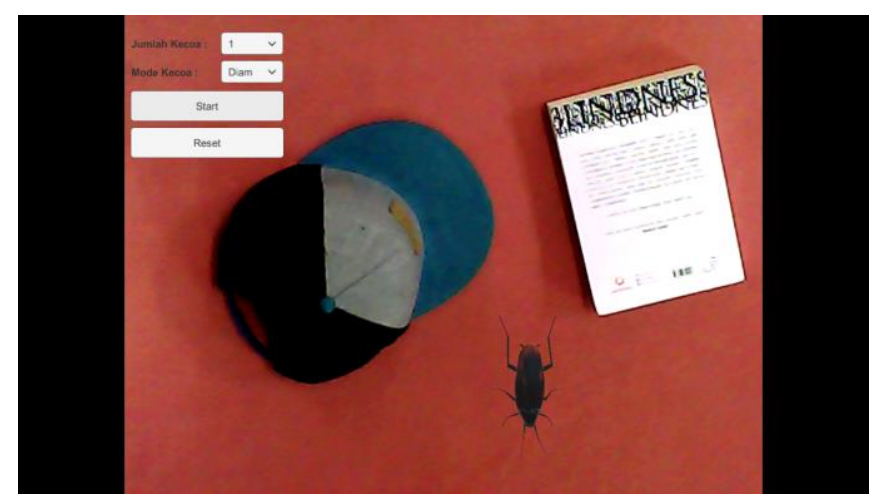

(b)

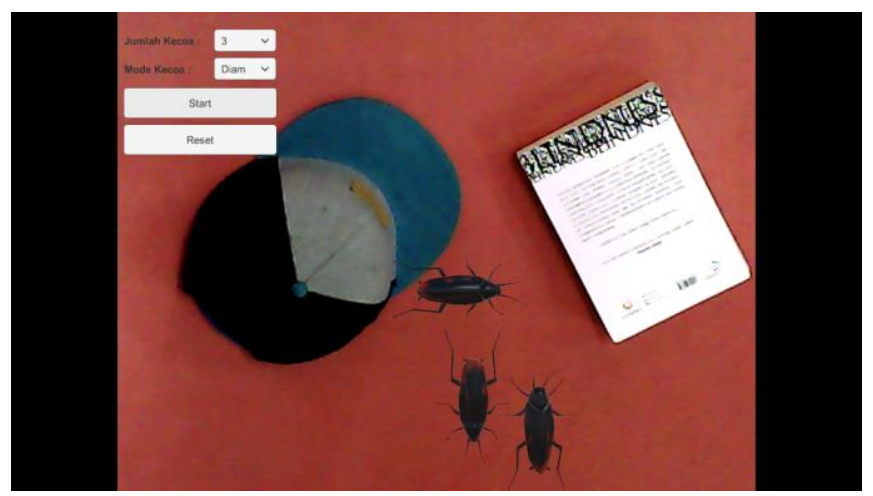

(c)

Fig. 1. (a) Testing environment (b) Scenario 1: one static cockroach (c) Scenario 3: three static cockroaches

moving cockroaches has the highest mean out of all of the scenarios with the highest score of 10 and the lowest is 1 . From these results, we can deduce that the higher the number of cockroaches, the higher the level of anxiety. While the moving mode receive higher score than the static mode.

For the sense of presence, Table 2 shows the result related to the presence measures. The highest score for question 1 regarding the sense of being of the participants is 7 from half of the participants while the lowest is 2 . This result shows that participants feel there were almost no gap in reality between
Table 2.

SUDs score for each scenario

\begin{tabular}{c|cccccccc}
\hline \hline Scenario & P1 & P2 & P3 & P4 & P5 & P6 & P7 & P8 \\
\hline $\boldsymbol{I}$ & 1 & 2 & 4 & 5 & 3 & 1 & 7 & 3 \\
\hline $\boldsymbol{I I}$ & 1 & 4 & 5 & 8 & 5 & 3 & 8 & 4 \\
\hline $\boldsymbol{I I I}$ & 3 & 3 & 5 & 6 & 5 & 7 & 8 & 2 \\
\hline $\boldsymbol{I} \boldsymbol{V}$ & 3 & 7 & 6 & 10 & 5 & 7 & 8 & 1
\end{tabular}

Table 3.

SUS score to measure the presence and reality judgment

\begin{tabular}{c|cccccccc}
\hline \hline Question & P1 & P2 & P3 & P4 & P5 & P6 & P7 & P8 \\
\hline $\boldsymbol{I}$ & 5 & 7 & 2 & 5 & 7 & 6 & 7 & 7 \\
\hline $\boldsymbol{I I}$ & 6 & 5 & 3 & 5 & 7 & 6 & 7 & 5 \\
\hline $\boldsymbol{I I I}$ & 4 & 4 & 2 & 5 & 7 & 6 & 6 & 4 \\
\hline $\boldsymbol{I} \boldsymbol{V}$ & 5 & 5 & 4 & 4 & 7 & 5 & 6 & 7 \\
\hline $\boldsymbol{V}$ & 5 & 5 & 4 & 4 & 6 & 6 & 7 & 6 \\
\hline $\boldsymbol{V I}$ & 5 & 6 & 4 & 5 & 5 & 5 & 7 & 7 \\
\hline
\end{tabular}

the testing environment and the environment shown on screen. Question 2 regarding the cockroach model shown throughout the procedure, has a highest score of 7 and a lowest score of 3 . For question 3, when the participants were asked whether they remember the cockroaches from the procedure as if they were real or not with 7 being a real one, one participant gave the highest score 7 while the lowest is 2 . In question 4, regarding the sense of being in the same room as if there was a real cockroach or not with 1 being not, has the highest score of 7 with the lowest score of 4 . The same as question 5 when asked about whether the scenarios felt like how it would have been in real life, the participants gave the highest score of 7 and the lowest score of 4 . For the last question, regarding whether the participants felt like in the same room with a real cockroach has the highest score of 7 and the lowest being 4. Overall, we can conclude based on this questionnaire that this application has a considerably good level of presence and reality.

\section{CONCLUSION}

In this paper, we have carried out a study with eight-phobic participants using a marker less augmented reality application for cockroach phobia. The results indicate that this application was able to induce a small to medium level of anxiety in most participants depending on their level of fear towards cockroaches. Meanwhile, the presence score was quite high with an existence of at least one of the highest score, 7 , for each questions and with the lowest score of 2 from participant 3 in two questions regarding the model of cockroaches and the study's testing environment.

In most cases, the higher the number of cockroaches the higher the anxiety level of the participants. As for the mode of cockroaches, the moving mode receive higher score compared to the static mode.

Most of the participants feel a small to high level of fear towards the possibility of cockroaches flying unto them. Further study can refer to this result in a form of a new scenario where the cockroach models fly towards the 
participants' direction.

The main shortcoming of this study is the small sample size. We need to apply the study to larger samples that includes a control group for a longer period assess the effectiveness of the application accurately. A certified physician also needed to conduct the initial assessment for a more reliable score. One possible future study would be to enhance the realism of this application by incorporating Oculus Rift technology.

\section{ACKNOWLEDGMENTS}

The author would like to thank Anny Yuniarti and Ridho Rahman Hariadi for their assistance in the development of this application.

\section{REFERENCES}

[1] R. J. Morris, T. R. Kratochwill, C. Sanders and S. Wiemer, "Fears and phobias in school age children," in Children's needs: Psychological perspectives, Washington, D.C., National Association of School Psychologists, 1987.

[2] G. L. Martin and J. Pear, Behavior Modification: What It Is and How To Do It, 7th ed., New Jersey: Pearson Education International, 2003.

[3] M. Smith, R. Segal and J. Segal, "Phobias and Fears: Symptoms, Treatment, and Self-Help for Phobias and Fears," 1999. [Online]. Available: http://www.helpguide.org/articles/anxiety/phobias-andfears.htm. [Accessed 18 December 2014].

[4] L. Kerawalla, R. Luckin, S. Seljeflot and W. Adrian, "“Making it real": Exploring the potential of augmented reality for teaching primary school science," Virtual Reality, vol. 10, pp. 163-74, 2006.

[5] R. Azuma, Y. Baillot, R. Behringer, S. Feiner, S. Julier, and B. MacIntyre, "Recent advances in augmented reality", Computers \& Graphics, vol. 25, pp. 1-15, 2001.

[6] R. T. Azuma, "A survey of augmented reality," Presence: Teleoperators \& Virtual Environments, vol. 6, pp. 355-85, 1997.

[7] P. J. Breton-Lopez, P. S. Quero, P. C. Botella, P. A. Garcia-Palacios, P. R. M. Banos and P. M. Alcaniz, "An Augmented Reality System Validation for the Treatment of Cockroach Phobia," Cyberpsychology, Behavior, and Social Networking, vol. 0, no. 0, 2010

[8] M. C. Juan, M. Alcaniz, C. Monserrat, C. Botella, R. M. Banos, and B. Guerrero. "Using Augmented Reality to Treat Phobias," IEEE Comput. Graph. vol. 25, no. 6, pp. 31-37, 2005.

[9] M. C. Juan and D. Joele, "A Comparative Study of The Sense of Presence and Anxiety in An Invisible Marker Versus A Marker Augmented Reality System for The Treatment of Phobia Towards Small Animals," vol. 69, no. 6, pp. 440-453, 2011.

[10] O. Libre, "Smokey Brown Cockroach Cycles,"Blend Swap, LLC., 17 May 2012. [Online]. Available: http://www.blendswap.com/blends/view/44633. [Accessed December 2014].

[11] S. Blackman, Beginning 3D Game Development with Unity: All-in-one, multi-platform game development, Apress, 2011.

[12] G. Bradski and A. Kaehler, Learning OpenCV: Computer Vision with the OpenCV Library, O'Reilly Media, 2008.

[13] Enox Software, "OpenCV for Unity," Enox Software, [Online]. Available: https://www.assetstore.unity3d.com/en/\#!/content/21088. [Accessed 23 May 2016].

[14] Weisstein, Eric W, "Central Moment." From MathWorld--A Wolfram Web Resource. http://mathworld.wolfram.com/CentralMoment.html.

[15] J. Szymanski and W. O'Donohue, "Fear of Spiders Questionnaire," Journal of Behavior Therapy and Experimental Psychiatry, vol. 26, no. 1, pp. 31-34, 1995.

[16] J. Wolpe, "The Practice of Behavior Therapy," New York, Pergamon
Press, 1969.

[17] M. Slater, M. Usoh and A. Steed, "Depth of presence in virtual," Presence-Teleoperators and Virtual Environments, vol. 3, no. 2, pp. 130-144, 1994. 